\title{
SOX2 and BRN2 as Key Transcription Factors in Neural Rosette Formation In Vitro
}

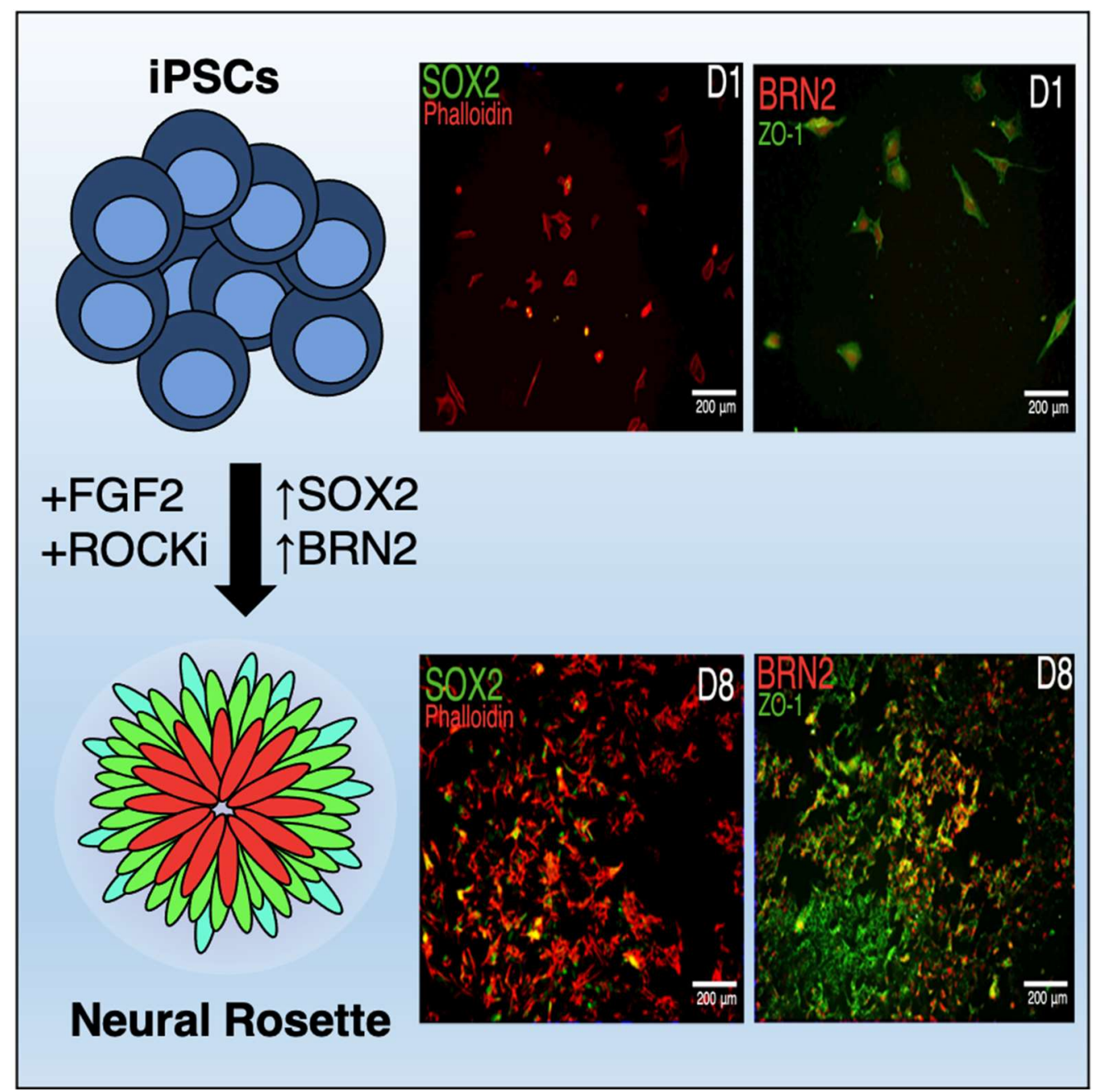

Author: Zuzana Hudáčová

https://doi.org/10.51892/ysm.1.202102

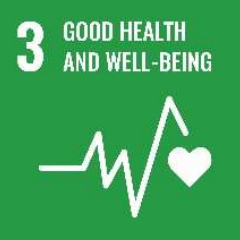




\title{
SOX2 and BRN2 as Key Transcription Factors in Neural Rosette Formation In Vitro
}

\author{
Zuzana Hudáčová 1 \\ ${ }^{1}$ Department of Histology and Embryology, Medical Faculty of Masaryk University, Czech Republic
}

\begin{abstract}
Although neurogenesis has been well studied, its molecular mechanisms remain largely unknown due to the challenges posed by the complexity of the underlying processes. Whilst in vivo studies can be used to study neurogenesis, the inability to control confounding variables complicate findings. Therefore, the purpose of this study was to identify the markers of in vitro neural rosette formation and describe the formation of neural rosettes from pluripotent stem cells using immunofluorescence analysis. The protocol of stem cell cultivation and induction of neural rosette formation was tested. Following, two transcription factors, BRN2 and SOX2, were fluorescently labelled and cells were imaged over a period of eight days. It was identified that SOX2 and BRN2 are expressed during in vitro neural rosette formation. These results are concurrent with in vivo neurogenesis, which suggests that neural rosettes could be a suitable in vitro model for researching neural development. Given that mistakes can arise during neurogenesis, such as neural tube defects, developing robust models to understand the formation of the nervous system is important. Moving forward, a detailed molecular understanding of neural rosette formation has the potential to be used for targeting specific transcription factors to treat or prevent problematic neurogenesis.
\end{abstract}

Keywords: BRN2, SOX2, transcription factors, neurogenesis, neural tube defects, induced pluripotent stem cells, in vitro, immunofluorescence, embryogenesis, differentiation 


\section{INTRODUCTION}

\section{In Vivo Neural Tube Formation - Neurogenesis}

During human development, the sperm and egg cell fusion results in a totipotent diploid zygote, which subsequently undergoes rapid mitotic divisions [1]. Once there are at least sixteen daughter cells, a ball-shaped structure known as a morula is formed. On the fifth day after fertilisation, the morula develops into a hollow ball of cells called a blastocyst [2]. In humans, a larger number of cells are concentrated at one pole of the blastocyst cavity: these cells are referred to as the inner cell mass (ICM; also known as the embryoblast). The remaining single-layer part of the cavity is referred to as the trophoblast and has a vital role in nutrition and nidation [3]. The cells of the ICM are characterised by their unique ability to give rise to all somatic cell types, which means that they are pluripotent and, when transferred to in vitro cultivation conditions, they can give rise to human embryonic stem cells [4].

Formation of the blastocyst is followed by gastrulation, whereby three foetal layers are formed: the endoderm and ectoderm are first to form, followed by the formation of the mesoderm [5]. As soon as the neurulation in the ectoderm occurs, specifically in the thickened plate stretched over the notochord, the embryo develops into a neurula. Neurulation is divided into primary and secondary stages which result in the formation of the nervous system.

During the process of primary neurulation, the ectoderm located directly over the notochord becomes divided into three sets of cells: internally positioned cells forming the neural plate, the externally positioned epidermis of the skin, and the neural crest cells that connect the neural plate and epidermis [6]. The lateral folding and bending of the neural plate then result in an elevation of two walls, known as the neural folds, that flank the neural groove's ventral midline floor plate [7]. The neural tube formation occurs when the two dorsolateral apical surfaces of the neural folds meet, fusing at the dorsal midline and separating from the overlying ectoderm [8]. Bends in the medial portion of each neural fold form into a tube-like structure so that the lumen of the newly formed neural tube remains intact as the neural folds converge.

Secondary neurulation is the process of caudal neural tube formation, which commences upon completion of primary neurulation [9]. This process is seen in the posterior region of the body in all vertebrates, including humans. Although secondary neurulation was histologically described more than half a century ago, its molecular mechanisms remain largely unknown [10]. During secondary neurulation, the medullary cord is formed, which later forms cavities that merge into a single tube [6]. In most of the observed vertebrates, secondary neurulation has been reported in the lumbar (abdominal) neural tube and tail vertebrae [11]. Secondary neurulation occurs in the presence of intact cutaneous ectoderm, overlying the caudal cell mass. Therefore, defects of secondary neurulation are considered to give rise to closed malformations [12].

In contrast to primary neurulation, secondary neurulation is somewhat disorganised, as several small "neural tubes" that are radially arranged around the central lumen are formed. Disordered secondary neurulation during prenatal development has been reported to result in several defects [10]. Therefore, understanding the molecular basis of neural tube formation, through modelling neurogenesis in vitro, has become increasingly 
important in developing therapeutics to target molecules involved in disordered neurulation.

\section{Stem Cells and In Vitro Models}

Stem cells, a unique type of undifferentiated cells, can differentiate into specialised cells needed for various tissues [13]. Fundamental properties of stem cells include differentiation and infinite self-renewal capabilities [14]. These properties make it possible to induce the differentiation of stem cells into various tissues using a specific type of cultivation in vitro. Several types of stem cells have been described to date, characterised by different properties, formation times, and origins. Embryonic stem cells (ESCs) are pluripotent stem cells derived from the ICM of a blastocyst [15]. ICM cells, still pluripotent at this stage, allow the isolated ESCs to maintain their pluripotent status and possess the ability to differentiate into any human body cells under specific in vitro conditions [16].

Adult stem cells can be found in various human tissues and play an essential role in the regeneration of damaged tissues wherein they reside. Adult stem cells are multipotent; their development depends on the extrinsic niche where the cell resides [17]. Somatic stem cells are more available for experimental purposes than ESCs. This is because ESC research is ethically and politically controversial since it involves the destruction of human embryos [18]. However, their smaller capacity to differentiate limits their usage in the research of in vitro neurogenesis [19]. The solution to this problem is induced pluripotent stem cells (iPSCs). Specialised adult cells of any tissue can be genetically reprogrammed with Yamanaka factors into iPSCs that exhibit essential ESC characteristics [20]. Due to their induced pluripotency - the theoretical ability to turn into any human cell - they come with various research and therapeutic benefits and have become a popular way to model neurogenesis.

\section{Neurulation Modelling from iPSCs In Vitro}

There are various methods used to induce neurulation in vitro, depending on the stage to be observed. Using the differentiation potential of iPSCs, the cultivation conditions can be modified so that the induced differentiation yields the desired cell types.

Previous research has shown that neuroectoderm induction requires the expression of epidermal transcription factors to be repressed, rather than neural differentiation to be stimulated. This suggests that the ectoderm's default state is neural [20]. In vitro neurogenesis models include the formation of so-called "embryoid bodies", spontaneously differentiating iPSCs in non-adherent cell culture conditions. These 3D spheroids recapitulate embryogenesis of different tissues originating from all three germ layers, including primitive neural tissue [21]. However, this approach is lengthy, with reduced control over the early phases of the process [22]. A commonly used technique in neurogenesis and modelling early neural development is the use of neurosphere formation assay, yet only a small percentage of cells within each neurosphere fulfil the criteria for being neural stem cells [23, 24]. This is because neurospheres each contain cells at multiple stages of differentiation; thus, they are not considered suitable for modelling early neural development.

Neurogenesis can also be modelled in 2D cell culture conditions via the induction of neural rosette formation. The neural rosette consists of neuroprogenitor cells that give rise to all neural and glial cell types [25]. When their differentiation is induced in embryonic stem 
cells' cultures, rosettes are radial arrangements of columnar cells, while they express proteins present in the neural tube [25]. Neurons, oligodendrocytes, and astrocytes are the three main classes of neuroepithelial cells that the neuroprogenitors in neural rosettes differentiate into in vivo [25]. The formation of neural rosettes has so far been described in pathological conditions, such as in teratomas. It has been suggested that neural rosettes represent disordered neural tube-like structures, resembling in vivo secondary neurulation, while they express a wide range of markers of neural precursors [26]. This suggests neural rosettes could be an important model for the understanding of not only neural tube formation, but also the emergence of neural tube defects.

\section{SOX2 and BRN2}

There are multiple transcription factors and proteins that play a role in the formation of neural rosettes. Fibroblast growth factor 2 (FGF2) belongs to the family of cellular signalling proteins necessary for normal development, thus it is often used in cell cultures [27]. Sex determining region Y-box 2 (SOX2) is a transcription factor known for its role during embryonic development as it contributes significantly to the regulation of cell pluripotency. Additionally, it is closely associated with early embryonic development, neural differentiation, and sexual differentiation [28]. It is also a critical factor for directing the differentiation of iPSCs to neural progenitors and for maintaining the properties of neural progenitor stem cells [29]. Brain-2 (BRN2) is also a transcription factor that is specific for neurogenesis [30]. Detecting the presence of both factors during in vitro neural rosette formation would indicate the similarity of this model with in vivo neurogenesis. Additionally, it would show that these proteins could possibly be targeted in the development of treatments during neural tube defects. Lastly, Zona occludens-1 tight junction protein (ZO-1) represents a protein that is specifically localised to the lumen of the newly forming rosettes, which is useful for the purpose of visualisation during microscopy [31].

Therefore, the aim of this study was to use in vitro immunofluorescence analysis of specific neural markers to map the neural rosette formation in vitro. The labelled biological molecules used to achieve the purpose of this study include proteins SOX2, filamentous actin (F-actin), BRN2, and ZO-1, while ZO-1 and Factin served the purpose of cell visualisation [32]. There are differential expressions of SOX2 and BRN2 during the formation of a neural rosette. The analysis of these transcription factors can add to the deeper molecular understanding of neural rosette formation. This could help identify how neural rosette formation resembles in vivo neurogenesis. It also helps with the potential use of neural rosettes as models, and the use of SOX2 and BRN2 as targets for developing treatments for neural tube defects.

\section{METHODS}

\section{iPSC Cultivation}

The differentiation was carried out in compliance with the neural rosette differentiation protocol (used in Bohačiaková Lab). Experiments involved iPSCs cultivation in a medium supporting the growth of undifferentiated stem cells. This medium contained fibroblast growth factor 2 (FGF2). For both cultivation and differentiation, a layer of Matrigel was used. Matrigel is a gel-like mixture of proteins that are used as substrates for culturing cells and resembles the extracellular environment in tissues [33]. Cell culture dishes were covered with Matrigel before being incubated at $4^{\circ} \mathrm{C}$ for at least one 
hour. Cell suspension was required to calculate the cells accurately and distribute them into cell culture dishes. Pre-prepared cells were first dissociated with the TrypLE express enzyme, trypsin, to create the desired single-cell suspension. A part of the well-mixed suspension was placed in a Bürker chamber, and the cells were counted. When using the Bürker chamber, a square section divided into nine smaller segments marked with a thick double line can be seen under the microscope. The cells were cultivated on Matrigel-covered cell culture dishes, the density being 2,500 cells $/ \mathrm{cm}^{2}$ in the presence of an hESC medium with FGF2, with $20 \mu \mathrm{M}$ of ROCKi (Rho-kinase inhibitor - to prevent apoptosis following cell dissociation). During the first day of differentiation, the medium was removed and replaced with a rosette differentiation medium containing ROCKi. From Day (D) 4 onwards, the rosette differentiation medium was changed once every 24 hours until neural rosettes appeared. Cells in different phases of neurodifferentiation were fixed using a $4 \%$ paraformaldehyde solution.

\section{Immunofluorescence Staining}

The indirect immunofluorescence staining (Fig. 1) was performed with mouse-generated primary antibodies against SOX2 and ZO-1. Rabbit-generated staining antibody BRN2 and phalloidin, which specifically binds to F-actin, was conjugated with fluorescence dye TRITC. Secondary staining antibodies SOX2 and ZO1 were conjugated with a green fluorochrome, while BRN2 was conjugated with red fluorochrome. To avoid unspecific binding of secondary antibody (that would result in nonspecific fluorescence signal), a blocking buffer (Tab. 2) containing PBS, 5\% of normal serum generated in the same species as the secondary antibody (goat), and $0.03 \%$ Triton $^{\mathrm{TM}}$ $\mathrm{X}-100$ (for cell membrane permeabilisation) were used. Antibodies were diluted in a buffer containing PBS, 1\% BSA (to block nonspecific areas), and $0.03 \%$ Triton $^{\mathrm{TM}} \mathrm{X}-100$. Cells were stained with HOECHST dye to visualise the nucleus, and MOWIOL was used as a mounting medium.

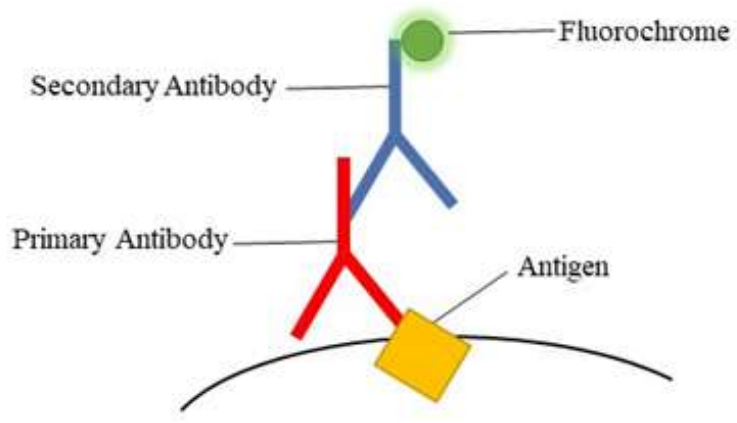

Figure 1 - Indirect immunofluorescence. Diagram constructed by Author based on Ref [35].

Following the immunofluorescence protocol used by Bohačiaková Lab [34], samples containing the individual stages of iPSC differentiation toward neuroectoderm, from D1 up to D8, were fixed and subsequently incubated in a blocking buffer. The prepared primary antibody was diluted in a dilution buffer (Tab. 3) at a 1:100 ratio. Once the buffer had been aspirated, the antibody in the buffer was added to the samples. Samples were incubated at $4^{\circ} \mathrm{C}$ overnight. The following day, samples were washed in PBS. Then, samples were incubated for one hour at room temperature with the secondary antibody conjugated with a fluorochrome diluted in the dilution buffer at 1:200. Cells were then washed with PBS, and cell nuclei were stained with HOECHST diluted in a dilution buffer at a 1:2000 ratio.

\section{Microscopy}

Slides containing the cells were mounted with MOWIOL and the mounting medium was left to harden overnight. The time points of differentiation were analysed using a Leica 500 fluorescence microscope. SOX2 and ZO-1 
were excited at the $488 \mathrm{~nm}$ range and detected around $517 \mathrm{~nm}$; BRN2 and Phalloidin were excited around $568 \mathrm{~nm}$ and detected around $603 \mathrm{~nm}$

\section{RESULTS}

\section{iPSC Cultivation}

The following cultivation protocol appeared to be successful, and the differentiation of iPSCs into neural rosettes was induced at around 7-8 days (Fig. 2). The formation of neural rosettes in vitro demonstrates a replicable protocol that can be used for modelling neurogenesis.

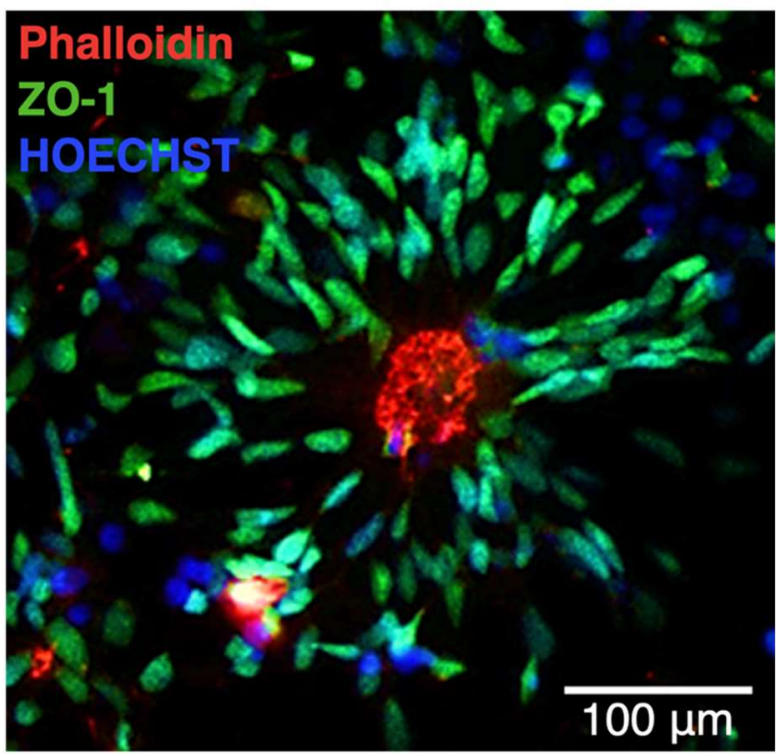

Figure 2 - Neural Rosette. Neural rosettes start to appear on D7 and D8.

\section{Analysis of iPSC Differentiation into Neural Rosettes}

Given that neural rosettes were formed, to understand how BRN2 and SOX2 are implicated during neural rosette formation, cells were labelled with primary and fluorescently tagged secondary antibodies against SOX2, BRN2 and ZO-1, as well as fluorescently conjugated phalloidin. Following this, they were imaged at different time points. D1 represented early stages of iPSC induction to neuroectoderm, resulting in a low cell distribution density. D1 revealed no significant expression of SOX2 protein (Fig. 3A), but SOX2 was more observable during D2 (see Fig. S1). Furthermore, generally, low expression of BRN2 protein was observed, suggesting SOX2 plays a more important role than BRN2 during the earlier stages of neural rosette formation. D2 and D3 represented the stages whereupon the first signs of cell grouping induction could be observed, with the cell grouping being even more visible on D4. D4 showed a higher consistency of cell distribution and an increased expression of the SOX2 protein accompanied by an increase in BRN2 expression (see Fig. S1). Together, these data reveal that during early in vitro neurogenesis, the onset of SOX2 expression comes sooner than the expression of BRN2, which appears only towards the end of early neural rosette formation. This shows that SOX2 plays a more important role than BRN2 during early in vitro neurodifferentiation.

On D5, a change in the organisation and consistency of cell distribution was observed (Fig. 3B) since the cells were grouped and more compact compared to D4. The density of cell distribution kept growing until D7, when the first signs of neural rosette formation could be observed (Fig. 3C), along with the positive signal of the SOX2 protein (which appeared to have the strongest signal during D3-5). The signal continued to disappear until D8 when neural rosettes could be observed (see Fig. S1, Fig. 2). The signal for BRN2 kept growing until $D 7$, suggesting BRN2 is more relevant for later stages of in vitro neurogenesis. Based on these results, it appears that BRN2 and SOX2 are not as strongly expressed once the neural rosettes are formed, implicating other proteins may be involved in maintaining the neural rosette stability. 

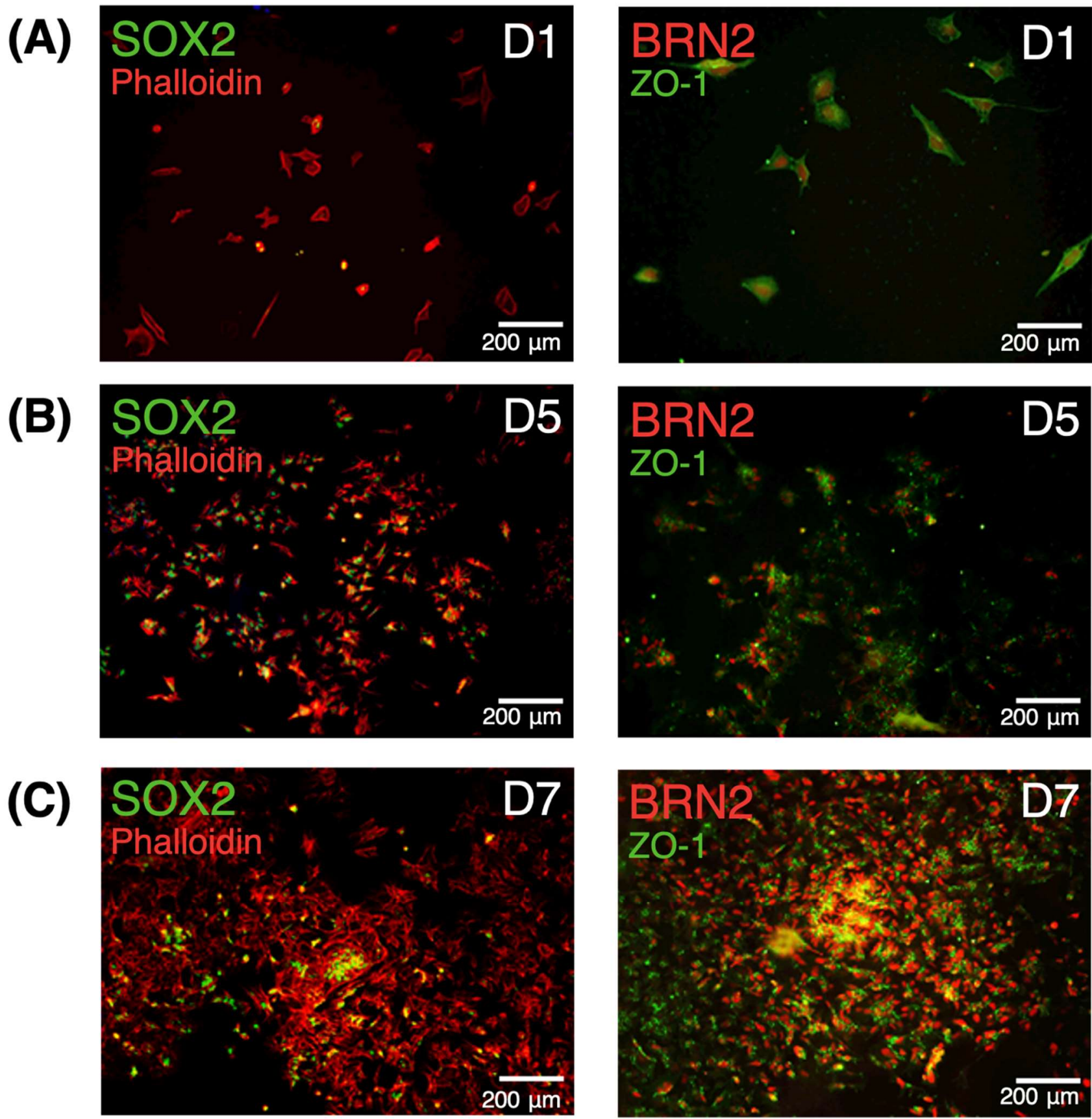

Figure 3 - Induced in vitro formation of neural rosettes from iPSCs. LHS: Cells were stained with SOX2 (green channel) and Phalloidin (red channel). RHS: Cells were stained with ZO-1 (green channel) and BRN2 (red channel). (A) D1. Cells in D1 are widely spread, expression of SOX2 and BRN2 is low. (B) D5. Cells are becoming more compact than on D4, high expression of SOX2, emerging expression of BRN2. (C) D7. Very high BRN2 signal, positive signal for SOX2. Neural rosettes are starting to form. 


\section{DISCUSSION}

The purpose of this experiment was to observe the formation of neural rosettes from iPSCs in vitro and understand the roles of the proteins BRN2 and SOX2 during their formation. The protocol induced the differentiation process and as expected, the formation of neural rosettes during the seventh and eighth days of differentiation. This corresponds with previous findings [36], where the formation of neural rosettes was observed during D7-D8. Therefore, the results obtained by indirect immunofluorescence (ZO-1 staining was used to visualise cellular tight junctions and protein F-actin to visualize the cytoskeleton and cell distribution of cells) visualised the formation of neural rosettes as expected [37].

Immunofluorescence analysis enabled studying the areas and times of expression of SOX2 and BRN2 proteins. It was observed that the expression of both proteins gradually increases in proportion to the time elapsed from the beginning of the induced differentiation process, with SOX2 being more prominent during earlier stages and BRN2 during later stages of neural rosette formation. This shows both SOX2 and BRN2 can be considered markers of in vitro neural rosette formation. These findings suggest that SOX2 and BRN2 are expressed during neurodifferentiation, supporting previous research stating that these components are involved in neural development [38]. Prior research indicates that rosettes most closely mimic the neural plate stage, which is a phase of early neural development [39]. As mentioned, the results of this study show the presence of SOX2 and BRN2, two markers of early neurogenesis, during neural rosette formation, thus they support the findings of Elkabetz et al., which suggest that neural rosettes reveal a functionally distinct early neural stem cell stage [39].
Immunofluorescence analysis was carried out in compliance with the protocol, which had been used and successfully implemented on a long-term basis. Research teams have used this method, for example, to study the inhibition of the Notch signalling pathway in hESCderived neural stem cells [40]. Researching neural development in vitro has the potential to develop innovative therapeutics to target specific transcription factors, using these models when researching neural tube defects.

The molecular process of differentiating iPSCs from D1 up to D8 was elucidated in this study (Fig. 2). The results showed that, based on the presence of two investigated protein markers during neural rosette differentiation, this model shows resemblance to an in vivo neurogenesis. This indicates that both SOX2 and BRN2 are prominent transcription factors during neurogenesis. However, there are other proteins that may play a particularly important role during this process. Future studies could involve selecting more known neurogenesisspecific protein markers, such as Notch signalling proteins [41], PAX6, SOX1, or nestin [42], to provide a more detailed description of the neural rosette formation and its comparison with neurodifferentiation during embryogenesis.

Both the visualisation of the tight junction proteins and cytoskeleton by staining the ZO-1 protein and staining using phalloidin, respectively, was successful, thus the spatial distribution of the cells could be observed. It was found that both SOX2 and BRN2 are markers of the in vitro differentiation of iPSCs into neural rosettes since their expression increased in proportion to the time from the beginning of the differentiation process, with SOX2 appearing earlier than BRN2. As both SOX2 and BRN2 are considered in vivo markers of neurodifferentiation, these results show the resemblance of the neural rosette 
model with in vivo neurogenesis. The implication behind these findings is that neural rosettes could potentially be used for pharmaceutical purposes, when researching and developing treatments for neural tube defects, by regulating the expression of transcription factors such as SOX2 and BRN2.

\section{ACKNOWLEDGEMENTS}

This study was part of a more comprehensive research initiative that is currently underway at the Masaryk University, at the Department of Histology and Embryology of the Medical Faculty. I would like to take this opportunity to thank Mgr. Dáša Bohačiaková, PhD, who trained me in lab processes, taught me what it takes to be a researcher, and helped me immensely to improve this study. I would also like to thank Assistant Professor MVDr. Aleš Hampl, CSc. for granting me his approval to work in his laboratories at the Masaryk University in Brno. I would like to express my gratitude to Bc. Veronika Fedorová who saved me numerous times during my lab struggles. Lastly, I would like to dedicate this work to my mother, who supported my scientific endeavours.

\section{AUTHOR'S NOTE}

All figures, or tables, were created by the Author, unless otherwise mentioned in the description provided of said figure.

\section{REFERENCES}

[1] C. De Paepe, M. Krivega, G. Cauffman, M. Geens and $\mathrm{H}$. Van de Helde, "Totipotency and lineage segregation in the human embryo," Molecular Human Reproduction, vol. 20, no. 7, pp. 599-618, 2014. Available: https://doi.org/10.1093/molehr/gau027.

[2] C. E. Boklage, How New Humans Are Made: Cells and Embryos, Twins and Chimeras, Left and Right,
Mind/Self/Soul, Sex, and Schizophrenia, London: World Scientific, 2009.

[3] M. Knöfler et al., "Human placenta and trophoblast development: key molecular mechanisms and model systems," Cellular and Molecular Life Sciences, vol. 76, no.18, pp. 3479-3496, 2019. Available: https://doi.org/10.1007/s00018-019-03104-6.

[4] J. A. Thomson et al., "Embryonic Stem Cell Lines Derived from Human Blastocysts," Science, pp. 11451147 , 1988. Available:

https://doi.org/10.1126/science.282.5391.1145.

[5] S. Ang and R. R. Behringer, "3 - Anterior-Posterior Patterning of the Mouse Body Axis at Gastrulation," Mouse Development: Patterning, Morphogenesis, and Organogenesis, pp. 37-53, 2002. Available: $\quad$ https://doi.org/10.1016/B978-0125979511/50005-6.

[6] S. F. Gilbert. Developmental Biology, 6th edition. Sunderland: Sinauer Associates, 2000.

[7] $\mathrm{Dr}$ T. P. Rothman, "Ectoderm: Neurulation, Neural Tube, Neural Crest," pp. 1-7. [Online]. Available: http://ftp.columbia.edu/itc/hs/medical/humand ev/2004/Chapt4-Ectoderm.pdf.

[8] E. Nikolopoulou, G. L. Galea, A. Rolo, N. D. E. Greene and A. J. Copp, "Neural tube closure: cellular, molecular and biomechanical mechanisms," Development, vol. 144, no. 4, pp. 552-566, 2017. Available: https://doi.org/10.1242/dev.145904.

[9] A. J. du Plessis and J. J. Volpe, "Chapter 1 - Neural Tube Development," Volpe's Neurology of the Newborn (Sixth Edition), pp. 3-33, 2018. Available: https://doi.org/10.1016/B978-0-323-428767.00001-6.

[10] Y. Takahashi and E. Shimokita, "Secondary neurulation: Another type of neurulation by mesenchymal-to-epithelial transition," Differentiation, vol. 80, pp. S6-S7, 2010. Available: https://doi.org/10.1016/j.diff.2010.09.146.

[11] L. A. Lowery and H. Sive, "Strategies of vertebrate neurulation and a re-evaluation of teleost neural tube formation," Mechanisms of Development, vol. 121, no.10, pp. 1189-1197, 2004. Available: https://doi.org/10.1016/j.mod.2004.04.022.

[12] A. J. Copp and N. D. E. Greene, "Neural tube defects--disorders of neurulation and related embryonic 
processes," Wiley Interdisciplinary Reviews: Developmental Biology, vol. 2, no. 2, pp. 213-227, 2012. Available: https://doi.org/10.1002/wdev.71.

[13] S. K. W. Oh and A. B. H. Choo, "1.23 - Stem Cells," Comprehensive Biotechnology (Third Edition), vol. 1, pp. 331-354, 2011. Available: https://doi.org/10.1016/B9780-444-64046-8.00021-5.

[14] J. K. Biehl and B. Russell, "Introduction to Stem Cell Therapy," Journal of Cardiovascular Nursing, vol. 24, no. 2. pp. 98-103, 2009. Available: https://dx.doi.org/10.1097/JCN.0b013e318197a6a5.

[15] J. Colas and G. C. Schoenwolf, "Towards a cellular and molecular understanding of neurulation," Developmental Dynamics, vol. 221, no. 2, pp. 117-145, 2001. Available: https://doi.org/10.1002/dvdy.1144.

[16] T. Vazin and W. J. Freed, "Human embryonic stem cells: Derivation, culture, and differentiation: A review," Restorative Neurology and Neuroscience, vol. 28, no. 4, pp. 589-603, 2010. Available: https://dx.doi.org/10.3233/RNN-2010-0543.

[17] G. Seydoux and R. E. Braun, "Pathway to Totipotency: Lessons from Germ Cells," Cell, vol. 127, no. 5, pp. 891-904, 2006. Available: https://doi.org/10.1016/j.cell.2006.11.016.

[18] B. Lo and L. Parham, "Ethical Issues in Stem Cell Research", Endocrine Reviews, vol. 30, no. 3, pp. 204213, 2009. Available: https://doi.org/10.1210/er.2008$\underline{0031}$.

[19] W. Zakrzewski, M. Dobrzyński, M. Szymonowicz and Z. Rybak, "Stem cells: past, present, and future," Stem Cell Research \& Therapy, vol. 10, no. 68, 2019. Available: https://doi.org/10.1186/s13287-019-1165-5.

[20] K. Takahasi and S. Yamanaka, "Induction of Pluripotent Stem Cells from Mouse Embryonic and Adult Fibroblast Cultures by Defined Factors," Cell, vol. 126, no. 4, pp. 663-676, 2006. Available: https://doi.org/10.1016/j.cell.2006.07.024.

[21] A. Leahy, J. Xiong, F. Kuhnert and H. Stuhlmann, "Use of developmental marker genes to define temporal and spatial patterns of differentiation during embryoid body formation," Journal of Experimental Zoology, vol. 284, no. 1, pp. 67-81, 1999. Available: https://doi.org/10.1002/(SICI)1097-

010X(19990615)284:1\%3C67::AIDJEZ10\%3E3.0.CO;2-O
[22] H. Azari and B. A. Reynolds, "In Vitro Models for Neurogenesis," Cold Spring Harbor Perspectives in Biology, vol. 8, no. 6, 2016. Available: https://dx.doi.org/10.1101/cshperspect.a0212 79.

[23] S. Mahendram, M. K. Subapanditha, N. McFarlane, C. Venugopal and S. K. Singh, "Chapter 17 - FlowCytometric Identification and Characterization of Neural Brain Tumor-Initiating Cells for Pathophysiological Study and Biomedical Applications," Neural Surface Antigens: From Basic Biology Towards Biomedical Applications, pp. 199-211, 2015. Available: https://doi.org/10.1016/B978-0-12-800781$\underline{\text { 5.00017-7. }}$.

[24] J. B. Jensen and M. Parmar, "Strengths and limitations of the neurosphere culture system," Molecular Neurobiology, vol. 34, no.3, pp. 153-161, 2006. Available: https://doi.org/10.1385/mn:34:3:153.

[25] P. G. Wilson and S. S. Stice, "Development and differentiation of neural rosettes derived from human embryonic stem cells," Stem Cell Reviews, vol. 2, no.1, pp. 66-77, $2006 . \quad$ Available: https://doi.org/10.1007/s12015-006-0011-1.

[26] S. C. Zhang, M. Wernig, I. D. Duncan, O. Brüstle and J. A. Thomson, "In vitro differentiation of transplantable neural precursors from human embryonic stem cells," Nature Biotechnology, vol. 19, pp. 1129-1133, 2001. Available: https://doi.org/10.1038/nbt1201-1129.

[27] L. M. Cotton, M. K. O'Bryan and B. T. Hinton, "Cellular Signaling by Fibroblast Growth Factors (FGFs) and Their Receptors (FGFRs) in Male Reproduction," Endocrine Reviews, vol. 29, no. 2, pp.193-216, 2008. Available: https://dx.doi.org/10.1210/er.2007-0028.

[28] J. Wang, H. Zeng, H. Li, J. Zhang and S. Wang, "Roles of sex-determining region $\mathrm{Y}$-box 2 in cell pluripotency and tumor-related signaling pathways (Review)," Molecular and Clinical Oncology, vol. 3, no. 6, pp.1203-1207,

2015.

Available: https://dx.doi.org/10.3892/mco.2015.639

[29] S. Zhang and W. Cui, "Sox2, a key factor in the regulation of pluripotency and neural differentiation," World Journal of Stem Cells, vol. 6, no. 3, pp. 305-311, 2014.

Available:

https://dx.doi.org/10.4252/wjsc.v6.i3.305.

[30] M. H. Dominiguez, A. E. Ayoub and P. Rakic, "POUIII Transcription Factors (Brn1, Brn2, and Oct6) Influence Neurogenesis, Molecular Identity, and Migratory Destination of Upper-Layer Cells of the Cerebral Cortex," 
Cerebral Cortex, vol. 23, no. 11, pp. 2632-2643, 2013. Available: https://doi.org/10.1093/cercor/bhs252.

[31] J. Medelnik et al., "Signaling-Dependent Control of Apical Membrane Size and Self-Renewal in RosetteStage Human Neuroepithelial Stem Cells," Stem Cell Reports, vol. 10, no. 6, pp. 1751-1765, 2018. Available: http://dx.doi.org/10.1016/j.stemcr.2018.04.018

[32] O. Tornavaca et al., "ZO-1 controls endothelial adherens junctions, cell-cell tension, angiogenesis, and barrier formation," Journal of Cell Biology, vol. 208, no. 6 , pp. 821-838, 2015.

Available: https://doi.org/10.1083/jcb.201404140.

[33] C. S. Hughes, L. M. Postovit and G. A. Lajoie, "Matrigel: A complex protein mixture required for optimal growth of cell culture," Proteomics, vol. 10, no. 9, pp. 1886-1890, 2010. Available: https://doi.org/10.1002/pmic.200900758.

[34] V. Fedorova et al., "Differentiation of neural rosettes from human pluripotent stem cells in vitro is sequentially regulated on a molecular level and accomplished by the mechanism reminiscent of secondary neurulation," Stem Cell Research, vol. 40, 2019. Available: https://doi.org/10.1016/j.scr.2019.101563.

[35] S. Joshi and D. Yu, "Chapter 8: Immunofluorescence," Basic Science Methods for Clinical Researchers, pp. 135-150, 2017. Available: https://doi.org/10.1016/B978-0-12-803077-6.00008-4.

[36] H. Hříbková, M. Grabiec, D. Klemová, I. Slaninová and $Y$. Sun, "Calcium signaling mediates five types of cell morphological changes to form neural rosettes," Journal of Cell Science, vol. 131, no. 3, 2018. Available: http://dx.doi.org/10.1242/jcs.206896.

[37] M. Itoh et al., "The 220-kD protein colocalizing with cadherins in non-epithelial cells is identical to ZO-1, a tight junction-associated protein in epithelial cells: cDNA cloning and immunoelectron microscopy," Journal of Cell Biology, vol. 121, no. 3, pp. 141-502, 1993. Available: https://doi.org/10.1083/jcb.121.3.491.

[38] T. C. Archer, J. Jin and E. S. Casey, "Interaction of Sox1, Sox2, Sox3 and Oct4 during primary neurogenesis," Developmental Biology, vol. 350, no. 2, pp. 429-440, $2011 . \quad$ Available: https://doi.org/10.1016/j.ydbio.2010.12.013.

[39] Y. Elkabetz et al., "Human ES cell-derived neural rosettes reveal a functionally distinct early neural stem cell stage," Genes \& Development, vol. 22, no. 2, pp. 152-
165, 2008.

Available: https://dx.doi.org/10.1101/gad.1616208.

[40] L. Borghese et al., "Inhibition of Notch Signaling in Human Embryonic Stem Cell-Derived Neural Stem Cells Delays G1/S Phase Transition and Accelerates Neuronal Differentiation In Vitro and In Vivo," Stem Cells, vol. 28, no. 5, pp. 955-964, 2010 Available: https://doi.org/10.1002/stem.408.

[41] H. Main, J. Radenkovic, S. Jin, U. Lendahl and E. R. Andersson, "Notch Signaling Maintains Neural Rosette Polarity," PLoS One, vol. 8, no. 5, 2013. Available: https://doi.org/10.1371/journal.pone.0062959.

[42] S. Malchenko et al., "Onset of rosette formation during spontaneous neural differentiation of hESC and hiPSC colonies," Gene, vol. 534, no. 2, pp. 400-407, 2014.

Available: https://doi.org/10.1016/i.gene.2013.07.101. 
YOUTH

3 GOOD HEALTH

Matters

ORIGINAL RESEARCH

SUPPLEMENTARY DATA

Table S1 - Rosette differentiation medium composition.

\begin{tabular}{|l|l|}
\hline Component & Concentration \\
\hline DMEM/F12 medium & $94 \%$ \\
\hline N2 & $1 \%$ \\
\hline B-27 & $1 \%$ \\
\hline Vitamin A (vit. A) & $1 \%$ \\
\hline Glutamax & $1 \%$ \\
\hline Non-essential amino acids & $1 \%$ \\
\hline Penicillin-streptomycin (P/S) & $1 \%$ \\
\hline
\end{tabular}

Table S2 - Blocking buffer composition.

\begin{tabular}{|l|l|}
\hline Component & Concentration \\
\hline $1^{*}$ Phosphate Buffered Saline & $94.7 \%$ \\
\hline Normal serum & $5 \%$ \\
\hline Triton $^{\mathrm{TM}} \mathrm{X}-100$ & $0.3 \%$ \\
\hline
\end{tabular}

Table S3 - Antibody dilution buffer composition.

\begin{tabular}{|l|l|}
\hline Component & Concentration \\
\hline $1^{*}$ Phosphate Buffered Saline & $97.8 \%$ \\
\hline BSA & $2 \%$ \\
\hline Triton $^{\mathrm{TM}} \mathrm{X}-100$ & $0.2 \%$ \\
\hline
\end{tabular}

Youth STEM Matters | August 2021 | Issue 1 | https://doi.org/10.51892/ysm.1.202102

13 
(A) SOX2

Phalloidin

D1 BRN2

ZO-1

D1

.

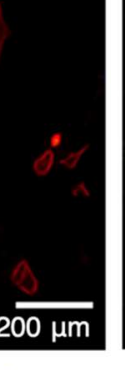

(B)

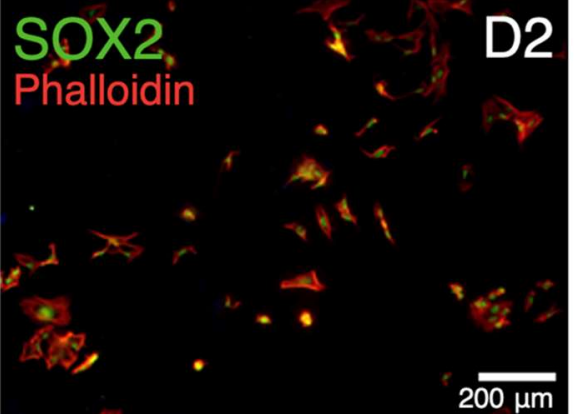

$\mathrm{BRN} 2$
$\mathrm{ZO}-1$

$200 \mu \mathrm{m}$

(B)

$200 \mu \mathrm{m}$

$-\infty$

(C)

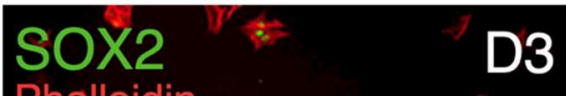

Phalloidin
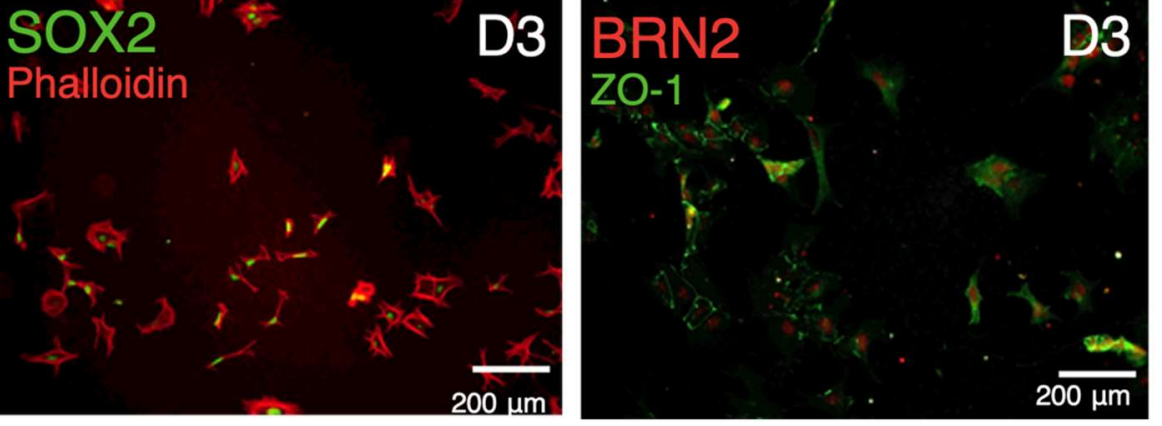

(D)
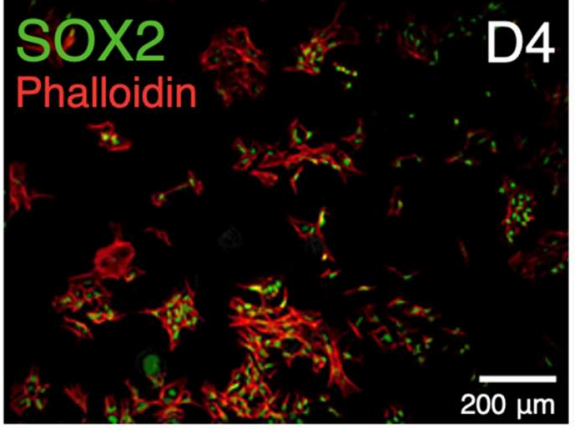
BRN2
ZO-1

D4

(E)
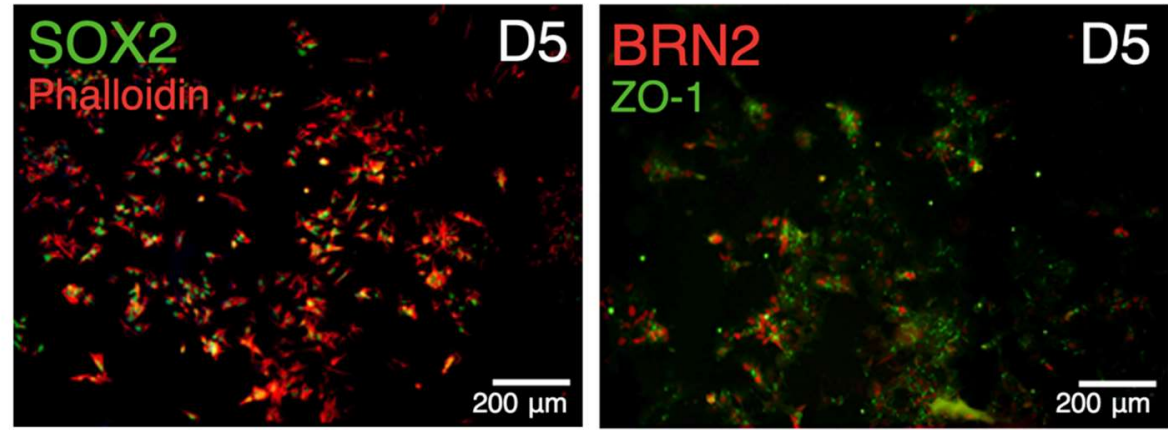
(F)
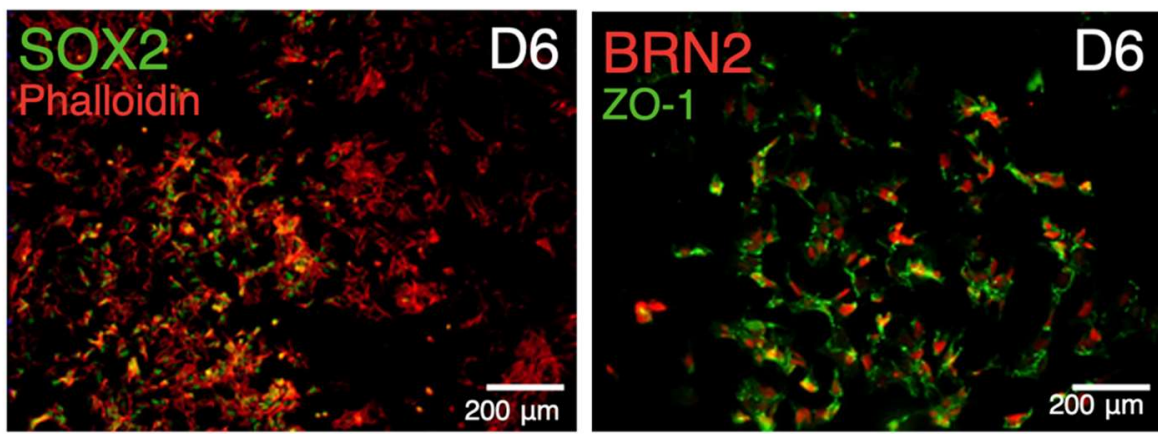

(G)
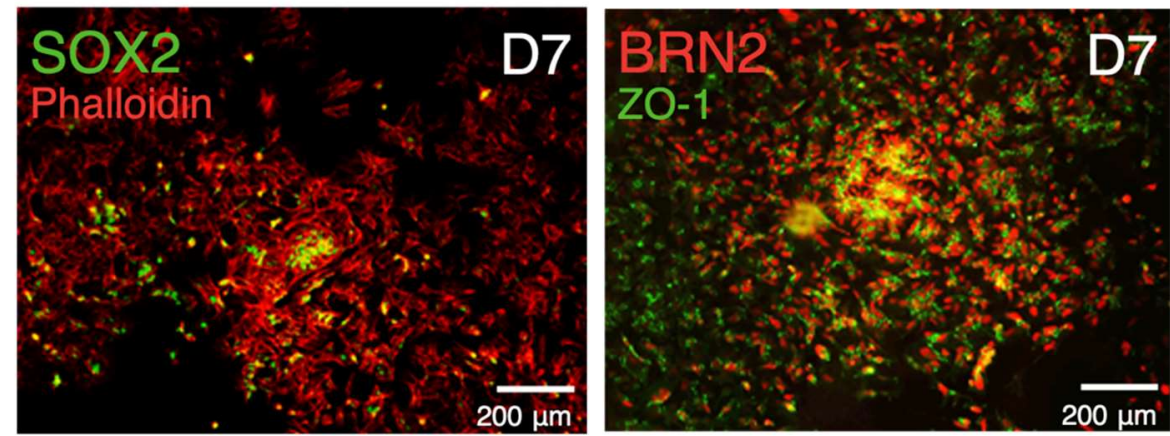

(H)
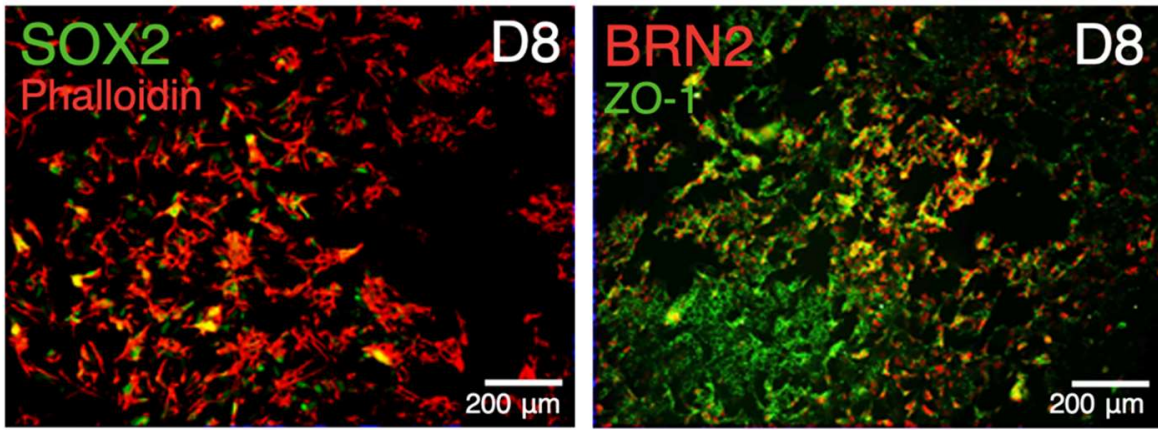

Figure S1 - Induced in vitro formation of neural rosettes from iPSCs. LHS: Cells were stained with SOX2 (green channel) and Phalloidin (red channel). RHS: Cells were stained with ZO-1 (green channel) and BRN2 (red channel). (A) D1. Cells in D1 are widely spread, expression of SOX2 and BRN2 is low. (B) D2. Low cell density, low BRN2 expression, emerging SOX2 expression. (C) D3. Cells are becoming more compact. (D) D4. An emerging expression of BRN2, higher SOX2 expression, higher cell grouping. (E) D5. Cells are becoming more compact than on D4, high expression of SOX2. (F) D6. Increased BRN2 expression. (G) D7. Very high BRN2 signal, positive signal for SOX2. Neural rosettes are starting to form. (H) D8. Lower SOX2 expression, and positive, yet lower, BRN2 signal. 